\title{
Componentes externos do corpo e gordura de descarte em vacas mestiças Charolês x Nelore abatidas com diferentes pesos ${ }^{1}$
}

\author{
Fernando Kuss ${ }^{2}$, João Restle ${ }^{3}$, Ivan Luiz Brondani ${ }^{4}$, Leonir Luiz Pascoal ${ }^{4}$, Luís Fernando \\ Glasenapp de Menezes ${ }^{5}$, Daniel Terra Leite ${ }^{6}$, Maurício Fernandes dos Santos ${ }^{6}$
}

\author{
1 Parte da Dissertação apresentada à UFSM pelo primeiro autor para obtenção do titulo de Mestre em Produção Animal. \\ 2 Doutorando do Programa de Pós-Graduação em Zootecnia da UFRGS. Bolsista CNPq. \\ 3 Professor visitante do CNPq - Departamento de Produção Animal - EV/UFG, Campus Samambaia, CP 131 - Goiânia - Go. \\ 4 Departamento de Zootecnia da UFSM. \\ ${ }^{5}$ Doutorando do Programa de Pós-Graduação em Zootecnia da UFSM \\ ${ }^{6}$ Mestrando do Programa de Pós-Graduação em Zootecnia da UFSM.
}

RESUMO - Objetivou-se estudar o desenvolvimento dos componentes externos e da gordura descartada durante o abate de vacas mestiças de segunda (G2 - 3/4Charolês (C) 1/4Nelore (N) e 3/4NC) e terceira gerações (G3 - 5/8CN e 5/8NC) do cruzamento rotativo Charolês $\times$ Nelore terminadas em confinamento e abatidas com 465 (T465), 507 (T507) ou $566 \mathrm{~kg}$ de PV (T566). Os animais apresentaram, em média, ao início do confinamento, 8,5 anos, 388,6 kg e 2,35 pontos de escore da condição corporal. A dieta alimentar, com relação volumoso:concentrado de 48:52, continha 12,5\% de PB e 2,99 Mcal de energia digestível por kg de MS. O peso de corpo vazio (PCV) (376,34, 412,53 e 463,64 kg, respectivamente) acompanhou o incremento do peso de abate (PA), porém, o rendimento de PCV não sofreu alteração e apresentou valor médio de $81,51 \%$ entre os grupos de abate. A elevação do PA e do PCV resultou em redução na participação do conteúdo da digesta. Houve maior desenvolvimento de cabeça e úbere nos animais mais pesados. Contudo, o desenvolvimento do couro permaneceu inalterado, o que resultou em menor participação relativa com o aumento do PA e do PCV. O aumento do peso de abate conferiu menor participação relativa do conjunto dos componentes externos no PCV $(16,47 ; 15,65$ e $14,30 \%$, respectivamente). A deposição de gordura interna se intensificou até o T507; a partir desse peso, aumentou a deposição de gordura subcutânea, ocorrendo maior remoção deste tecido na toalete das carcaças no T566 (4,02, 4,26 e 7,31 kg, respectivamente para T466, T507 e T566). Maior participação de Charolês no genótipo conferiu maior desenvolvimento da cabeça. Vacas da terceira geração do cruzamento rotativo Charolês $\times$ Nelore apresentaram maior tamanho de cabeça em relação às da segunda geração $(16,22$ vs $15,77 \mathrm{~kg})$.

Palavras-chave: bovinos de corte, couro, cruzamento, fêmeas, membros, tecido adiposo

\section{Body external components and discard fat of feedlot finished Charolais $\mathrm{x}$ Nellore crossbred cows slaughtered with different weights}

\begin{abstract}
The objective of this experiment was to study the development of external body components and that of discarded fat in feedlot finished crossbred cows from the second (G2 - 3/4Charolais (C) 1/4Nellore (N) and 3/4NC) and third $(\mathrm{G} 3-5 / 8 \mathrm{CN}$ and $5 / 8 \mathrm{NC})$ generations of a Charolais $\times$ Nellore rotational crossbreeding program slaughtered with 465 (T465), 507 (T507) and $566 \mathrm{~kg}$ (T566) of body weight. At the beginning of the trial, age, body weight and body condition score averaged, respectively, 8.5 years, $388.6 \mathrm{~kg}$ and 2.35 points. Animals were fed a diet with a forage to concentrate ratio of 48:52, which contained $12.5 \%$ of CP and 2.99 Mcal of digestible energy per kg of DM. The increment in empty body weight (EBW) (376.34, 412.53 e $463.64 \mathrm{~kg}$, respectively, for T465, T507 and T566) followed that of slaughter weight (SW) but EBW yield did not change and averaged $81.51 \%$ across the different SW groups. Increased SW and EBW reduced the proportion of digesta content. Animals with heavier body weight showed greater development of head and udder. However, skin growth was not affected leading to lower relative proportion with the increase of SW and EBW. Increasing SW also resulted in lower relative participation in the EBW of the external components (16.47, 15.65 and 14.30\%). Deposition of internal fat increased until T507 while that of subcutaneous fat was greatest on T566, resulting in larger removal of trim fat from carcasses of T566 (4.02, 4.26 and $7.31 \mathrm{~kg}$, respectively) cows. Greater participation of Charolais in the genotype resulted in higher head development. Cows of the third generation of the rotational crossbreeding Charolês $\times$ Nellore had greater head size than those from the second generation (16.22 against $15.77 \mathrm{~kg})$.
\end{abstract}

Key Words: beef cattle, crossbreeding, fat tissue, females, limbs, skin 


\section{Introdução}

Nos últimos anos, tem-se enfatizado o estudo dos componentes não-carcaça, como conseqüência da valorização dos órgãos aptos ao consumo, do couro e dos resíduos usados pela indústria de rações e, principalmente, por esses componentes estarem associados ao rendimento de carcaça; quando são mais pesados, o rendimento tende a ser menor, diminuindo o valor da carcaça.

No Brasil, ainda são escassas as pesquisas relacionadas ao desenvolvimento dos componentes não-carcaça em bovinos de corte, principalmente se consideradas a ampla composição genética do rebanho, as práticas de manejo, o sexo e a categoria animal. Pesquisas indicam diferenças no consumo alimentar (Galvão et al., 1991a; Moletta \& Restle 1992; Restle et al., 2000) e no rendimento de carcaça (Perobelli et al., 1995; Restle et al., 2002; Menezes et al., 2005 ) de bovinos Bos indicus e Bos taurus. Essas diferenças podem estar relacionadas, em parte, às características anatômicas do trato digestivo, ao peso dos tecidos de revestimento e aos membros.

Pesquisas sobre a participação de membros externos e de órgãos de revestimento em animais mestiços zebuínos apresentaram resultados divergentes (Galvão et al., 1991b; Fernandes et al., 2002), em decorrência da finalidade produtiva dos animais envolvidos no acasalamento (corte ou leite). Além do fator genético, verifica-se influência do estádio de maturidade sobre o desenvolvimento dos membros. Restle et al. (2005) constataram em novilhos abatidos aos 22 meses de idade redução da participação de cabeça e patas e valores similares para a participação do couro em relação ao corpo vazio com o aumento do peso de abate.

A quantidade de tecido adiposo depositado no corpo dos animais condiciona sua eficiência alimentar, pois este tecido, em comparação ao tecido muscular, requer maior quantidade de nutrientes para sua deposição. Além disso, segundo Thompson et al. (1983), o local de deposição do tecido adiposo no corpo altera as exigências nutricionais do animal. Esses autores demonstraram que a atividade metabólica do tecido adiposo na cavidade interna é superior à da gordura subcutânea, ocasionando, conseqüentemente, diferenças na exigência de energia para mantença desses animais. Solis et al. (1988) observaram menor exigência de mantença para vacas Brahman em relação a vacas taurinas, o que atribuíram, em parte, à menor deposição de gordura interna e à menor atividade metabólica dos órgãos internos desses animais.

A maioria dos resultados observados na literatura sobre os componentes não-carcaça foi gerada a partir de experimentos com machos em crescimento. No entanto, segundo o ANUALPEC (2005), aproximadamente 50\% dos animais destinados ao abate no Brasil são fêmeas de descarte. São escassos os estudos sobre o comportamento biométrico dos componentes externos (cabeça, membros e couro) de animais desta categoria, os quais podem representar aproximadamente $15 \%$ do valor pago pela carcaça.

Nesse contexto, realizou-se este estudo para avaliar o desenvolvimento biométrico dos componentes externos e a deposição de gordura corporal em vacas de descarte mestiças Charolês $\times$ Nelore abatidas com diferentes pesos.

\section{Material e Métodos}

O estudo foi realizado no Setor de Bovinocultura de Corte do Departamento de Zootecnia da Universidade Federal de Santa Maria, em Santa Maria - RS.

Foi estudado o desenvolvimento dos componentes externos e da gordura descartada durante o abate de vacas da segunda (G2 - 3/4Charolês (C) 1/4Nelore (N) e 3/4NC) e terceira gerações ( $\mathrm{G} 3-5 / 8 \mathrm{CN}$ e $5 / 8 \mathrm{NC})$ do cruzamento alternado Charolês $\times$ Nelore terminadas em confinamento e abatidas com 465 (T465), 507 (T507) ou $566 \mathrm{~kg}$ (T566) de peso vivo.

Foram utilizadas 24 vacas de descarte tomadas ao acaso do mesmo rebanho, sendo 12 animais da G2 (seis vacas $3 / 4 \mathrm{C}-1 / 4 \mathrm{~N}$ e seis vacas $3 / 4 \mathrm{~N}-1 / 4 \mathrm{C}$ ) e 12 da G3 (seis vacas $5 / 8 \mathrm{C}-3 / 8 \mathrm{~N}$ e seis vacas $5 / 8 \mathrm{~N}-3 / 8 \mathrm{C}$ ). Ao início do período experimental, os animais apresentavam, em média, 8,5 anos de idade, $388,6 \mathrm{~kg}$ de $\mathrm{PV}$ e 2,35 pontos de escore da condição corporal, segundo classificação proposta por Restle (1972), em que 1 = muito magro, 2 = magro, 3 = médio, $4=$ gordo e $5=$ muito gordo.

Durante o confinamento, os animais foram alimentados com a mesma dieta contendo $12,5 \%$ de PB e 2,99 Mcal de energia digestível $/ \mathrm{kg}$ de MS, composta de $48 \%$ de volumoso (silagem de milho - AG 5011) e 52\% de concentrado, composto de $92,6 \%$ de farelo de trigo, $6,8 \%$ de calcário calcítico e $0,6 \%$ de sal comum, com base na MS. Os animais foram alimentados duas vezes ao dia, às $9 \mathrm{e} 16 \mathrm{~h}$, mantendo-se oferta diária de MS 10\% superior ao consumo voluntário.

À medida que os lotes dos tratamentos atingiram, em média, o peso pré-determinado de abate, os animais foram submetidos a jejum de sólidos (12 horas), sendo pesados e transportados em caminhão boiadeiro por $25 \mathrm{~km}$ até o frigorífico comercial, onde foram abatidos logo após o desembarque. $\mathrm{O}$ abate foi realizado segundo o fluxo de abate normal do estabelecimento, sendo considerado peso de abate o peso final tomado na fazenda. 
Durante o abate, foram separados da carcaça e pesados os seguintes componentes: sangue; cabeça (com orelhas e chifres), pés, vassoura da cauda, couro, úbere (denominados componentes externos); coração, rins, fígado, baço e pulmões (denominados órgãos vitais); estômago (rúmen-retículo, abomaso e omaso) cheio e vazio, intestino (delgado e grosso) cheio e vazio (denominados trato gastrintestinal); gordura das vísceras, do estômago e dos órgãos (denominada gordura interna); e gordura de toalete. A soma das gorduras interna e de toalete representou a gordura total descartada.

Para obtenção do peso corporal vazio, utilizou-se o somatório dos pesos de carcaça quente, sangue, componentes externos, órgãos vitais, trato gastrintestinal vazio e gordura total.

O delineamento experimental utilizado foi o inteiramente casualizado, com arranjo fatorial $3 \times 2 \times 2$ (pesos de abate $\times$ gerações de cruzamento $\times$ grupos genéticos aninhados dentro de cada geração de cruzamento), sendo os dados submetidos à análise de normalidade pelo teste Shaphirowilk e à análise de variância pelo teste $\mathrm{F}$.

O modelo estatístico utilizado foi o seguinte:

$$
\begin{aligned}
& \mathrm{Y}_{\mathrm{ijkl}}=\mu+\mathrm{PA}_{\mathrm{i}}+\mathrm{G}_{\mathrm{j}}+\mathrm{GG}_{\mathrm{K}}\left(\mathrm{G}_{\mathrm{J}}\right)+(\mathrm{PA} * \mathrm{GG})_{\mathrm{ik}}+(\mathrm{PA} * \mathrm{G})_{\mathrm{ij}}+ \\
& \mathrm{PI}_{1}+\mathrm{E}_{\mathrm{ijk} 1}
\end{aligned}
$$

em que: $\mathrm{Y}_{\mathrm{ijkl}}=$ variáveis dependentes; $\mu=$ média de todas as observações; $\mathrm{PA}_{\mathrm{i}}=$ efeito do peso de abate de ordem $\mathrm{i}$; $\mathrm{G}_{\mathrm{j}}=$ efeito da geração de cruzamento de ordem $\mathrm{j} ; \mathrm{GG}_{\mathrm{K}}\left(\mathrm{G}_{\mathrm{J}}\right)=$ efeito do grupo genético de ordem $\mathrm{k}$, aninhado dentro da geração de cruzamento de ordem j; $(\mathrm{PA} * \mathrm{GG})_{\mathrm{ik}}=$ efeito da interação i-ésimo peso de abate $\times$ k-ésimo grupo genético; $\left(P A^{*} \mathrm{G}\right)_{\mathrm{ij}}=$ efeito da interação i-ésimo peso de abate $\times$ j-ésima geração de cruzamento; $\mathrm{PI}_{1}=$ efeito da covariável peso inicial de ordem 1; $\mathrm{E}_{\mathrm{ijkl}}=$ efeito aleatório residual.

As interações peso de abate $\times$ geração de cruzamento e peso de abate $\times$ grupo genético dentro de geração foram inicialmente testadas e removidas do modelo estatístico final, pois não foram significativas para as variáveis avaliadas. As médias das variáveis dependentes significativamente afetadas pelas variáveis independentes, segundo análise de variância, foram comparadas pelo teste de probabilidade da diferença (pdiff), a 10\% de significância, utilizando-se o pacote estatístico SAS (1997).

Realizou-se, ainda, a análise de contraste entre animais de predominância Charolês $(\mathrm{PC}=3 / 4 \mathrm{CN}+5 / 8 \mathrm{CN})$ e Nelore $(\mathrm{PN}=3 / 4 \mathrm{NC}+5 / 8 \mathrm{CN})$.

\section{Resultados e Discussão}

Não houve efeito significativo das interações peso de abate $\times$ geração de cruzamento e peso de abate $\times$ grupo genético da vaca sobre as variáveis estudadas e, portanto, são apresentadas e discutidas separadamente.

$\mathrm{Na}$ Tabela 1, verifica-se que os pesos de abate (PA) e de corpo vazio (PCV) apresentaram ritmo similar de crescimento entre os pesos extremos $(21,7$ e $23,2 \%$, respectivamente). Esses aumentos foram inferiores ao incremento do peso da carcaça quente (30\%), representado pelos tecidos muscular e adiposo, visto que, em termos absolutos, o tecido ósseo não sofreu alteração (Kuss et al., 2005b).

$\mathrm{Na}$ Tabela 2 constam os resultados referentes ao desenvolvimento de cabeça, pés, vassoura da cauda, úbere, couro e conjunto desses componentes externos (CCE).

Verificou-se maior desenvolvimento de cabeça no T566, o que pode estar associado à deposição dos tecidos muscular e adiposo neste membro, pois não houve desenvolvimento ósseo na carcaça dos animais com o aumento do peso (Kuss et al., 2005b). No entanto, quando esta variável foi corrigida para PA e PCV, os valores foram similares e inferiores nos pesos de abate mais elevados. Comportamento similar foi observado por Restle et al. (2005), que reportaram decréscimo linear da participação da cabeça no PA e PCV com o aumento do peso de abate de novilhos jovens com 22 meses de idade.

Aumento do peso de cabeça com a progressão do peso de abate foi descrito por Jorge \& Fontes (2001) e Signoretti et al. (1999), que, no entanto, não apontaram as

Tabela 1 - Peso e rendimento de corpo vazio (CV) e conteúdo de digesta de vacas mestiças Charolês $\times$ Nelore abatidas com diferentes pesos

Table 1 - $\quad$ Means and standard errors for empty body weight (EBW) and yield and digesta content according to slaughter weight

\begin{tabular}{lccc}
\hline $\begin{array}{l}\text { Característica } \\
\text { Trait }\end{array}$ & \multicolumn{3}{c}{$\begin{array}{c}\text { Peso de abate } \\
\text { Slaughter weight }\end{array}$} \\
\cline { 2 - 4 } & T465 & T507 & T566 \\
\hline
\end{tabular}

Peso de corpo $376,34 \pm 11,17 \mathrm{c} \quad 412,53 \pm 11,20 \mathrm{~b} \quad 463,64 \pm 11,18 \mathrm{a}$ vazio (PCV), $\mathrm{kg}$

Empty body weight

$(E B W)^{*}, k g$

Rendimento de $\quad 81,27 \pm 1,49 \quad 81,32 \pm 1,49 \quad 81,94 \pm 1,49$

corpo vazio, \%

Empty body weight

yield, \%

Conteúdo de $\quad 61,51 \pm 3,26 \quad 55,02 \pm 3,27 \quad 58,16 \pm 3,27$

digesta, $\mathrm{kg}$

Digesta content, $\mathrm{kg}$

Conteúdo de $\quad 13,24 \pm 0,71 \mathrm{a} \quad 10,86 \pm 0,72 \mathrm{~b} \quad 10,26 \pm 0,71 \mathrm{~b}$ digesta, $\%$ do PA

Digesta content*,

\% of $P A$

Conteúdo de $\quad 16,32 \pm 0,97 \mathrm{a} \quad 13,44 \pm 0,97 \mathrm{~b} \quad 12,58 \pm 0,97 \mathrm{~b}$

digesta, \% do PCV

Digesta content*,

$\%$ of $E B W$

$a, b, c$ : médias na mesma linha diferem $(P<0,05)$ pelo teste $t$. $a, b, c$ : means in the same row differ $(P<0.05)$ by $t$ test. 
Tabela 2 - Médias e erros-padrão para cabeça, pés, vassoura da cauda, úbere, couro e conjunto dos componentes externos (cabeça + pés + vassoura da cauda + úbere + couro) (CCE) de vacas mestiças Charolês $\times$ Nelore abatidas com diferentes pesos

Table 2 - Means and standard errors for head, feet, tail, udder, skin, external components (head + feet + tail + udder + skin) (CCE) according to slaughter weight

\begin{tabular}{|c|c|c|c|}
\hline \multirow[t]{2}{*}{$\begin{array}{l}\text { Característica } \\
\text { Trai }\end{array}$} & \multicolumn{3}{|c|}{$\begin{array}{l}\text { Peso de abate } \\
\text { Slaughter weight }\end{array}$} \\
\hline & T465 & Т5 07 & Т 566 \\
\hline & \multicolumn{3}{|c|}{$\begin{array}{l}\text { Peso absoluto, } \mathrm{kg} \\
\text { Absolut weight, } \mathrm{kg}\end{array}$} \\
\hline $\begin{array}{l}\text { Cabeça* } \\
\text { Head }\end{array}$ & $15,75 \pm 0,20 b$ & $15,62 \pm 0,20 \mathrm{~b}$ & $16,58 \pm 0,20 \mathrm{a}$ \\
\hline $\begin{array}{l}\text { Pés** } \\
\text { Feet }\end{array}$ & $7,07 \pm 0,26 b$ & $7,76 \pm 0,26 a$ & $7,21 \pm 0,26 b$ \\
\hline $\begin{array}{l}\text { Vassoura da cauda* } \\
\text { Tail }\end{array}$ & $0,30 \pm 0,03 b$ & $0,29 \pm 0,03 b$ & $0,42 \pm 0,03 a$ \\
\hline $\begin{array}{l}\text { Úbere* } \\
\text { Udder }\end{array}$ & $2,53 \pm 0,30 \mathrm{c}$ & $3,53 \pm 0,30 \mathrm{~b}$ & $4,74 \pm 0,30 \mathrm{a}$ \\
\hline $\begin{array}{l}\text { Couro } \\
\text { Skin }\end{array}$ & $35,58 \pm 1,63$ & $36,97 \pm 1,63$ & $37,08 \pm 1,63$ \\
\hline \multirow{2}{*}{$\begin{array}{l}\text { CCE** } \\
\text { CCE }\end{array}$} & $61,24 \pm 1,81 b$ & $64,17 \pm 1,82 \mathrm{ab}$ & $66,03 \pm 1,81 \mathrm{a}$ \\
\hline & \multicolumn{3}{|c|}{$\begin{array}{l}\% \text { do peso de abate } \\
\% \text { of slaughter weight }\end{array}$} \\
\hline $\begin{array}{l}\text { Cabeça** } \\
\text { Head** }\end{array}$ & $3,41 \pm 0,07 a$ & $3,10 \pm 0,07 b$ & $2,95 \pm 0,07 b$ \\
\hline $\begin{array}{l}\text { Pés** } \\
\text { Feet }\end{array}$ & $1,55 \pm 0,06 a$ & $1,53 \pm 0,06 a$ & $1,28 \pm 0,06 b$ \\
\hline $\begin{array}{l}\text { Vassoura da cauda } \\
\text { Tail }\end{array}$ & $0,06 \pm 0,01$ & $0,06 \pm 0,01$ & $0,07 \pm 0,01$ \\
\hline $\begin{array}{l}\text { Úbere* } \\
\text { Udder }\end{array}$ & $0,54 \pm 0,06 \mathrm{c}$ & $0,70 \pm 0,06 b$ & $0,85 \pm 0,06 a$ \\
\hline $\begin{array}{l}\text { Couro** } \\
\text { Skin }\end{array}$ & $7,86 \pm 0,35 a$ & $7,31 \pm 0,35 \mathrm{ab}$ & $6,59 \pm 0,35 b$ \\
\hline $\mathrm{CCE}^{* *}$ & $13,43 \pm 0,42 \mathrm{a}$ & $12,71 \pm 0,42 \mathrm{ab}$ & $11,73 \pm 0,42 b$ \\
\hline & \multicolumn{3}{|c|}{$\begin{array}{l}\% \text { do corpo vazio } \\
\% \text { of empty body }\end{array}$} \\
\hline $\begin{array}{l}\text { Cabeça* } \\
\text { Head }\end{array}$ & $4,20 \pm 0,11 \mathrm{a}$ & $3,82 \pm 0,11 b$ & $3,60 \pm 0,11 b$ \\
\hline $\begin{array}{l}\text { Pés* } \\
\text { Feet }\end{array}$ & $1,90 \pm 0,08 \mathrm{a}$ & $1,89 \pm 0,08 \mathrm{a}$ & $1,56 \pm 0,08 b$ \\
\hline $\begin{array}{l}\text { Vassoura da cauda } \\
\text { Tail }\end{array}$ & $0,08 \pm 0,01$ & $0,07 \pm 0,01$ & $0,09 \pm 0,01$ \\
\hline $\begin{array}{l}\text { Úbere** } \\
\text { Udder }\end{array}$ & $0,67 \pm 0,07 \mathrm{c}$ & $0,86 \pm 0,07 b$ & $1,03 \pm 0,07 a$ \\
\hline $\begin{array}{l}\text { Couro** } \\
\text { Skin }\end{array}$ & $9,62 \pm 0,37 \mathrm{a}$ & $9,01 \pm 0,37 \mathrm{ab}$ & $8,02 \pm 0,37 b$ \\
\hline $\begin{array}{l}\mathrm{CCE}^{* *} \\
\mathrm{CCE}\end{array}$ & $16,47 \pm 0,48 a$ & $15,65 \pm 0,48 a$ & $14,30 \pm 0,48 b$ \\
\hline
\end{tabular}

a,b,c: na mesma linha diferem ${ }^{* *}(P<0,05)$ ou * $(P<0, C C E)$ pelo teste $t$. $a, b, c$ : in the same row differ ${ }^{* *}(P<0.05)$ or ${ }^{*}(P<0.10)$ by t test.

possíveis causas para o fato. Além disso, Di Marco (1998) relatou que o peso da cabeça também pode ser afetado pelo sistema de alimentação, sendo menor em animais alimentados com concentrado em comparação àqueles alimentados com forragem.
O peso do úbere e sua participação no PA e PCV aumentaram gradativamente com o incremento do peso de abate dos animais, demonstrando correlação positiva e significativa entre estas características (Tabela 4). Essa tendência foi principalmente condicionada pela participação de tecido adiposo, visto que é intensa a deposição de gordura neste órgão. O peso do úbere esteve positivamente associado $(0,68)$ ao aumento da gordura total descartada (Tabela 4).

Apesar de o peso vivo entre os pesos extremos ter aumentado $101 \mathrm{~kg}$, não houve diferença significativa para o peso do couro, sendo constatado decréscimo em sua participação relativa no PA e no PCV (Tabela 2). Com a maior deposição de massa corporal, era previsível a ocorrência de aumento do peso do tecido de revestimento, o que não ocorreu, provavelmente em virtude da maior elasticidade deste componente com o aumento do peso, pois os animais eram de maturidade avançada. Entretanto, em novilhos jovens, ainda em fase de crescimento, o aumento de peso

Tabela 3 - Médias e erros-padrão para as gorduras de toalete interna e total descartada em vacas mestiças Charolês $\times$ Nelore abatidas com diferentes pesos

Table 3 - Means and standard errors for trim, internal and total discarded fat according to slaughter weight

\begin{tabular}{|c|c|c|c|}
\hline \multirow[t]{2}{*}{$\begin{array}{l}\text { Característica } \\
\text { Trait }\end{array}$} & \multicolumn{3}{|c|}{$\begin{array}{l}\text { Peso de abate } \\
\text { Slaughter weight }\end{array}$} \\
\hline & T465 & Т 507 & T566 \\
\hline & \multicolumn{3}{|c|}{$\begin{array}{l}\text { Peso absoluto, } \mathrm{kg} \\
\text { Absolute weight, } \mathrm{kg}\end{array}$} \\
\hline $\begin{array}{l}\text { Gordura de toalete } \\
\text { Trim fat }\end{array}$ & $4,02 \pm 0,49 b$ & $4,26 \pm 0,49 b$ & $7,31 \pm 0,49 a$ \\
\hline $\begin{array}{l}\text { Gordura interna } \\
\text { Internal fat }\end{array}$ & $15,97 \pm 1,83 b$ & $24,00 \pm 1,83 a$ & $28,16 \pm 1,83 a$ \\
\hline
\end{tabular}
descartada Total discarded fat

$\%$ do peso de abate

$\%$ of slaughter weight

Gordura de toalete $0,87 \pm 0,09 \mathrm{~b} \quad 0,82 \pm 0,09 \mathrm{~b} \quad 1,30 \pm 0,09 \mathrm{a}$ Trim fat

Gordura interna $\quad 3,47 \pm 0,28 b \quad 4,71 \pm 0,28 \mathrm{a} \quad 4,93 \pm 0,28 \mathrm{a}$

Internal fat

Gordura total $\quad 4,34 \pm 0,33 b \quad 5,53 \pm 0,33 a \quad 6,23 \pm 0,33 a$

descartada

Total discarded fat

$\%$ do corpo vazio

$\%$ of empty body

Gordura de toalete $1,07 \pm 0,10 \mathrm{~b} \quad 1,00 \pm 0,10 \mathrm{~b} \quad 1,58 \pm 0,10 \mathrm{a}$

Trim fat

Gordura interna $\quad 4,26 \pm 0,31 \mathrm{~b} \quad 5,78 \pm 0,31 \mathrm{a} \quad 6,01 \pm 0,31 \mathrm{a}$

Internal fat

Gordura total $\quad 5,33 \pm 0,34 \mathrm{~b} \quad 6,78 \pm 0,34 \mathrm{a} \quad 7,59 \pm 0,34 \mathrm{a}$

descartada

Total discarded fat

a,b,c: na mesma linha diferem $(P<0,05)$ pelo teste $t$.

$a, b, c:$ in the same row differ $(P<0.05)$ by t test. 


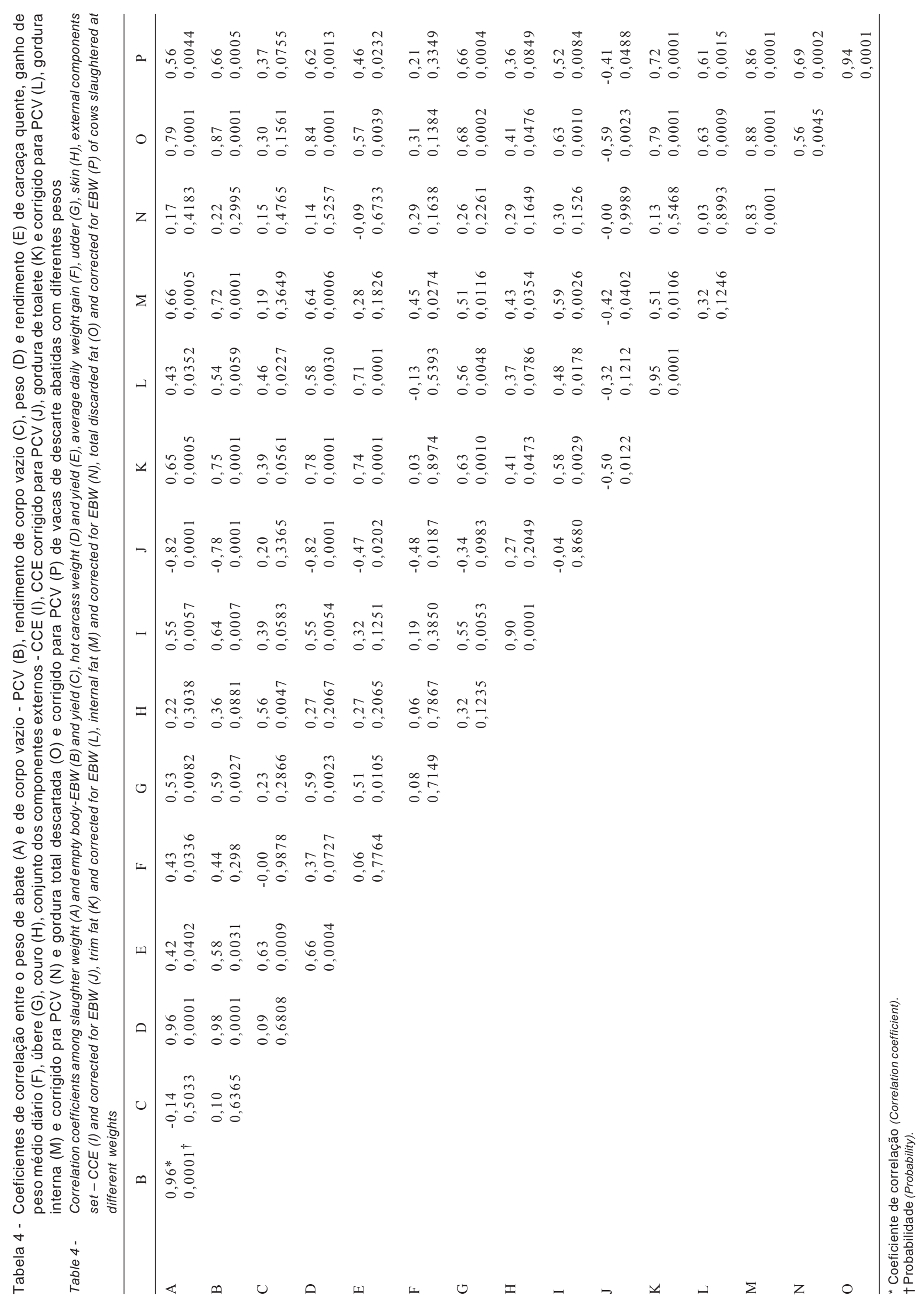


corporal resulta em incremento linear no peso de couro (Jessé et al., 1976; Jorge \& Fontes, 2001; Restle et al., 2005), de modo que a participação do couro no corpo vazio pode variar de $9,62 \%$ no T465 a $8,02 \%$ no T507. Esses valores estão de acordo com a afirmativa de Di Marco (1998) de que o peso do couro representa de 9 a $10 \%$ do peso vazio em bovinos e pode variar entre os biótipos dos animais. Maior participação do couro no PA e PCV com o avanço do estádio de maturidade de bovinos foi registrado por Restle et al. (2005), que verificaram média de 8,75 e $9,96 \%$, respectivamente.

Quando os componentes externos foram somados (CCE), os animais do T566 apresentaram maior valor absoluto em relação ao T465 e T507. Porém, quando expressos em valor relativo ao PA e PCV, ocorreu decréscimo desses componentes no peso de abate mais elevado, o que explica, em parte, o maior rendimento de carcaça no T566 (Kuss et al., 2005a) e a correlação negativa entre a participação do CCE no PCV e o peso $(-0,82)$ e o rendimento de carcaça quente $(-0,47)$, demonstrando que a maior participação relativa desses componentes no corpo vazio resulta em decréscimo do peso e do rendimento de carcaça quente.

Os resultados reportados na literatura não são unânimes quanto ao desenvolvimento desses componentes. Galvão et al. (1991b) reportaram maior participação de CCE no PCV em animais mais leves ao abaterem novilhos com peso equivalente a 90,100 e $110 \%$ do peso à idade adulta das matrizes $(18,67 ; 16,95$ e $17,40 \%$, respectivamente). Restle et al. (2005), no entanto, em estudo com novilhos abatidos aos 425, 467 e 510 kg, não observaram diferença significativa nos pesos desses componentes em relação ao PCV (média de $15,83 \%)$. Os resultados encontrados neste estudo dife- rem dos valores reportados por Di Marco (1998), que citou variação de 15 a $17 \%$ de CCE no PCV de bovinos.

Em valores absolutos, a gordura interna sofreu incremento de $50,3 \%$ do T465 para o T507, mantendo similar até o T566 (Tabela 3). Quando corrigido para PA e PCV, verificou-se a mesma tendência, o que indica a prioridade inicial de deposição de gordura na cavidade interna dos animais do T465 ao T507. A partir deste período até o T566, houve maior deposição de gordura subcutânea $(4,72 ; 4,40$ e 7,54 mm, respectivamente, para T465, T507 e T566; Kuss et al., 2005a), condicionando à maior quantidade de gordura de toalete (Tabela 3 ). Os valores obtidos para espessura de gordura subcutânea, em relação ao PA, foram de 0,010 ; 0,009 e 0,013 mm e, em relação ao PCV, foram de 0,$012 ; 0,011$ e $0,016 \mathrm{~mm}$, respectivamente, para T465, T507 e T566. A deposição de gordura subcutânea intensificou a partir do T507, diferente da gordura interna, que foi maior do T460 ao T507, o que está de acordo com a afirmativa de Di Marco (1998), que relatou que a deposição de gordura se inicia na região intermuscular, depois ocorre nas vísceras e na região subcutânea e, por fim, na região intramuscular.

A gordura total descartada expressa em relação ao PCV indicou que este tecido acompanhou o desenvolvimento do corpo vazio, demonstrando o potencial de ganho corporal energético das vacas de descarte com o aumento do peso. Mesmo comportamento foi observado por Leme et al. (2000) e Restle et al. (2005), que verificaram aumento linear desta fração com o aumento do peso de abate em novilhos jovens. Almeida et al. (2001) descreveram em seu estudo que o conteúdo de proteína no ganho de peso do corpo vazio reduziu e os teores de gordura e energia aumentaram com o aumento do peso de corpo

Tabela 5 - Médias e erros-padrão para peso de abate (PA), peso (PCV) e rendimento de corpo vazio (RCV), conteúdo de digesta (DIG), percentual de digesta no peso de abate (DIGPA) e percentual de digesta no PCV (DIGPCV) de acordo com a geração de cruzamento (G2 e G3) e o grupo genético (GG)

Table 5 - $\quad$ Means and standard errors for slaughter weight (SW), empty body weight (EBW) and yield (EBWY), digesta content (DIG), digesta proportion in relation to slaughter weight (DIGSW) and digesta percentage of empty body (DIGEBW) according to crossbreeding generation (G2 and G3) and genetic group (GG)

\begin{tabular}{|c|c|c|c|c|c|c|}
\hline GG & $\begin{array}{c}\text { PA, kg } \\
S W\end{array}$ & $\begin{array}{c}\mathrm{PCV}, \mathrm{kg} \\
E B W\end{array}$ & $\begin{array}{c}\mathrm{RCV}, \% \\
E B W Y\end{array}$ & $\begin{array}{c}\text { DIG, } \mathrm{kg} \\
\text { DIG }\end{array}$ & $\begin{array}{c}\text { DIGPA, \% } \\
\text { DIGSW }\end{array}$ & $\begin{array}{c}\text { DIGPCV, \% } \\
\text { DIGEBW }\end{array}$ \\
\hline $3 / 4 \mathrm{CN}$ & $515,02 \pm 12,21$ & $417,45 \pm 13,17$ & $81,26 \pm 1,23$ & $64,62 \pm 3,84$ & $12,47 \pm 0,84$ & $15,51 \pm 1,14$ \\
\hline $3 / 4 \mathrm{NC}$ & $492,11 \pm 13,04$ & $404,90 \pm 14,07$ & $82,26 \pm 1,32$ & $54,79 \pm 3,84$ & $11,37 \pm 0,90$ & $13,92 \pm 1,22$ \\
\hline G2 & $503,57 \pm 8,56$ & $411,18 \pm 9,24$ & $81,71 \pm 0,87$ & $59,71 \pm 2,70$ & $11,92 \pm 0,59$ & $14,72 \pm 0,80$ \\
\hline $5 / 8 \mathrm{CN}$ & $519,51 \pm 12,01$ & $421,01 \pm 12,96$ & $81,07 \pm 1,21$ & $54,87 \pm 3,78$ & $10,58 \pm 0,83$ & $13,05 \pm 1,12$ \\
\hline $5 / 8 \mathrm{NC}$ & $523,51 \pm 12,06$ & $426,65 \pm 13,00$ & $81,56 \pm 1,22$ & $58,64 \pm 3,80$ & $11,40 \pm 0,83$ & $13,96 \pm 1,33$ \\
\hline G3 & $521,51 \pm 8,56$ & $423,83 \pm 9,24$ & $81,31 \pm 0,87$ & $56,76 \pm 2,70$ & $10,99 \pm 0,59$ & $13,50 \pm 0,80$ \\
\hline
\end{tabular}

Diferença ${ }^{1}$

Difference ${ }^{1}$

$\mathrm{PC}-\mathrm{PN}$

9,45

3,44

$-0,74$

3,03

0,13

0,34

$(P>0,05) ;{ }^{1}$ Contraste entre predominância PC $(3 / 4 C N+5 / 8 C N)$ e $P N(3 / 4 N C+5 / 8 N C)$

$(P>0.05) ;{ }^{1}$ Contrast between $P C(3 / 4 C N+5 / 8 C N)$ and $P N(3 / 4 N C+5 / 8 N C)$ predominance. 
vazio de novilhos mestiços em ganho compensatório abatidos a cada $50 \mathrm{~kg}$ de PV (150 a $450 \mathrm{~kg}$ ).

Na comparação entre gerações de cruzamento e grupos genéticos, não houve diferenças significativas nas características dependentes avaliadas (Tabela 5). Utilizando novilhos não-castrados no sistema superjovem, Santos et al. (2003) não observaram alterações no PCV entre animais C e 3/4CN. Galvão et al. (1991b), no entanto, relataram que os animais Nelores apresentam maior rendimento de corpo vazio (RCV) em relação aos FI Nelore $\times$ Marchigiana (M) e
Nelore $\times$ Limousine $(\mathrm{L})$ e atribuíram esse resultado à heterozigose dos animais F1 Bos taurus $\times$ Bos indicus. No estudo de Jones et al. (1985), a diferença no RCV foi mais expressiva entre animais de corte de porte grande e animais de raças leiteiras.

Os animais $3 / 4 \mathrm{CN}$ apresentaram maior peso de cabeça $(16,59 \mathrm{~kg})$ em relação aos $3 / 4 \mathrm{NC}(14,91 \mathrm{~kg})$, o que pode ser justificado pela maior participação de sangue Charolês nos animais desse genótipo (Tabela 6). Comparando a média dos animais, aqueles com predominância de

Tabela 6 - Médias e erros-padrão para peso de cabeça, pés, vassoura da cauda, úbere, couro e conjunto dos componentes externos (cabeça + pés + vassoura da cauda + úbere + couro) (CCE) de acordo com a geração de cruzamento (G2 e G3) e o grupo genético (GG)

Table 6 - Means and standard errors for weight of head, feet, tail, udder, skin, external components set (head + feet + tail + udder + skin) (CCE) according to crossbreeding generation (G2 and G3) and genetic group (GG)

\begin{tabular}{|c|c|c|c|c|c|c|}
\hline $\begin{array}{l}\text { Grupo genético } \\
\text { Genetic group }\end{array}$ & $\begin{array}{c}\text { Cabeça } \\
\text { Head }\end{array}$ & $\begin{array}{l}\text { Pés } \\
\text { Feet }\end{array}$ & $\begin{array}{c}\text { Vassoura da cauda } \\
\text { Tail }\end{array}$ & $\begin{array}{l}\text { Úbere } \\
\text { Udder }\end{array}$ & $\begin{array}{l}\text { Couro } \\
\text { Skin }\end{array}$ & $\begin{array}{l}\text { CCE } \\
C C E\end{array}$ \\
\hline \multicolumn{7}{|c|}{$\begin{array}{c}\text { Peso absoluto, } \mathrm{kg} \\
\text { Absolut weight, } \mathrm{kg}\end{array}$} \\
\hline $3 / 4 \mathrm{CN}$ & $16,59 \pm 0,24 a$ & $7,58 \pm 0,30$ & $0,33 \pm 0,04$ & $3,99 \pm 0,36$ & $37,67 \pm 1,92$ & $66,16 \pm 2,14$ \\
\hline $3 / 4 \mathrm{NC}$ & $14,91 \pm 0,26 \mathrm{~b}$ & $6,95 \pm 0,33$ & $0,39 \pm 0,04$ & $3,14 \pm 0,38$ & $38,00 \pm 2,04$ & $63,39 \pm 2,28$ \\
\hline G2 & $15,75 \pm 0,17 \mathrm{~B}$ & $7,26 \pm 0,21$ & $0,36 \pm 0,03$ & $3,56 \pm 0,25$ & $37,84 \pm 1,34 \mathrm{~A}$ & $64,77 \pm 1,50$ \\
\hline $5 / 8 \mathrm{CN}$ & $16,37 \pm 0,24$ & $7,56 \pm 0,30$ & $0,35 \pm 0,04$ & $3,07 \pm 0,35 b$ & $33,52 \pm 1,89$ & $60,87 \pm 2,10$ \\
\hline $5 / 8 \mathrm{NC}$ & $16,07 \pm 0,24$ & $7,31 \pm 0,30$ & $0,28 \pm 0,04$ & $4,21 \pm 0,35 a$ & $36,97 \pm 1,89$ & $64,83 \pm 2,11$ \\
\hline G3 & $16,22 \pm 0,17 \mathrm{~A}$ & $7,43 \pm 0,21$ & $0,32 \pm 0,03$ & $3,64 \pm 0,25$ & $35,25 \pm 1,34 \mathrm{~B}$ & $62,85 \pm 1,50$ \\
\hline
\end{tabular}

Diferença ${ }^{1}$

Difference $^{1}$

\begin{tabular}{|c|c|c|c|c|c|c|}
\hline $\mathrm{PC}-\mathrm{PN}$ & $0,99 *$ & $0,44 *$ & 0,00 & $-0,15$ & $-1,89$ & $-0,59$ \\
\hline & \multicolumn{5}{|c|}{$\begin{array}{l}\% \text { do peso de abate } \\
\% \text { of slaughter weight }\end{array}$} & \\
\hline $3 / 4 \mathrm{CN}$ & $3,22 \pm 0,08$ & $1,47 \pm 0,08$ & $0,06 \pm 0,01$ & $0,77 \pm 0,41$ & $7,33 \pm 0,41$ & $12,86 \pm 0,50$ \\
\hline $3 / 4 \mathrm{NC}$ & $3,11 \pm 0,09$ & $1,46 \pm 0,08$ & $0,08 \pm 0,01$ & $0,64 \pm 0,07$ & $7,98 \pm 0,44$ & $13,27 \pm 0,53$ \\
\hline G2 & $3,17 \pm 0,06$ & $1,47 \pm 0,05$ & $0,07 \pm 0,01$ & $0,71 \pm 0,05$ & $7,66 \pm 0,29 \mathrm{C}$ & $13,06 \pm 0,35 \mathrm{C}$ \\
\hline $5 / 8 \mathrm{CN}$ & $3,18 \pm 0,08$ & $1,47 \pm 0,07$ & $0,07 \pm 0,01$ & $0,59 \pm 0,07 \mathrm{~d}$ & $6,56 \pm 0,40$ & $11,86 \pm 0,49$ \\
\hline $5 / 8 \mathrm{NC}$ & $3,11 \pm 0,08$ & $1,41 \pm 0,07$ & $0,05 \pm 0,01$ & $0,79 \pm 0,07 \mathrm{c}$ & $7,14 \pm 0,40$ & $12,50 \pm 0,49$ \\
\hline G3 & $3,14 \pm 0,06$ & $1,44 \pm 0,05$ & $0,06 \pm 0,01$ & $0,69 \pm 0,05$ & $6,85 \pm 0,29 \mathrm{D}$ & $12,18 \pm 0,35 \mathrm{D}$ \\
\hline
\end{tabular}

Diferença ${ }^{1}$

Difference ${ }^{1}$

\begin{tabular}{|c|c|c|c|c|c|c|}
\hline $\mathrm{PC}-\mathrm{PN}$ & 0,09 & 0,03 & 0,00 & $-0,03$ & $-0,61$ & $-0,52$ \\
\hline \multicolumn{7}{|c|}{$\begin{array}{l}\% \text { do corpo vazio } \\
\% \text { of empty body }\end{array}$} \\
\hline $3 / 4 \mathrm{NC}$ & $3,79 \pm 0,13$ & $1,78 \pm 0,10$ & $0,10 \pm 0,01$ & $0,78 \pm 0,08$ & $9,69 \pm 0,47$ & $16,13 \pm 0,57$ \\
\hline G2 & $3,88 \pm 0,09$ & $1,80 \pm 0,07$ & $0,09 \pm 0,01$ & $0,86 \pm 0,06$ & $9,35 \pm 0,31 \mathrm{~A}$ & $15,98 \pm 0,38 \mathrm{~A}$ \\
\hline $5 / 8 \mathrm{CN}$ & $3,92 \pm 0,12$ & $1,81 \pm 0,09$ & $0,08 \pm 0,01$ & $0,72 \pm 0,08 b$ & $8,09 \pm 0,43$ & $14,63 \pm 0,53$ \\
\hline
\end{tabular}

Diferença $^{1}$

Difference $^{1}$

PC - PN

0,15

0,06

0,00

$-0,04$

$-0,66$

$-0,49$

* $(P<0,10) ; a, b$ e $A, B$ : na mesma coluna diferem $(P<0,05)$ pelo teste $t$ entre grupo genético e geração de cruzamento, respectivamente.

c, d e C, D: na mesma coluna diferem $(P<0,10)$ pelo teste t entre grupo genético e geração de cruzamento, respectivamente.

${ }^{1}$ Contraste entre predominância PC $(3 / 4 C N+5 / 8 C N)$ e PN $(3 / 4 N C+5 / 8 N C)$.

* $(P<0.10) ; a, b$ and $A, B$; in same column differ $(P<0.05)$ by ' $t$ ' test between genetic group and crossbreeding generation, respectively.

$c, d$ and $C, D$ : in same column differ $(P<0.10)$ by 't' test between genetic group and crossbreeding generation, respectively.

${ }^{1}$ Contrast between $P C(3 / 4 C N+5 / 8 C N)$ and $P N(3 / 4 N C+5 / 8 N C)$ predominance. 
Tabela 7 - Médias e erros-padrão para as gorduras de toalete, interna e total descartada de acordo com a geração de cruzamento (G2 e G3) e o grupo genético (GG)

Table 7 - Means and standard errors for trim, internal and total discarded fat, according to crossbreeding generation (G2 and G3) and genetic group (GG)

\begin{tabular}{lccc}
\hline GG & $\begin{array}{c}\text { Gordura de toalete } \\
\text { Trim fat }\end{array}$ & $\begin{array}{c}\text { Gordura interna } \\
\text { Internal fat }\end{array}$ & $\begin{array}{c}\text { Gordura total } \\
\text { descartada } \\
\text { Total discarded fat }\end{array}$ \\
\hline \multicolumn{4}{c}{$\begin{array}{c}\text { Peso absoluto, kg } \\
\text { Absolute weight, kg }\end{array}$} \\
& \multicolumn{4}{c}{$20,92 \pm 2,15$} & $26,15 \pm 2,47$ \\
3/4CN & $5,23 \pm 0,57$ & $21,81 \pm 2,30$ & $26,63 \pm 2,64$ \\
3/4NC & $4,82 \pm 0,61$ & $21,36 \pm 1,51$ & $26,39 \pm 1,73$ \\
G2 & $5,03 \pm 0,40$ & $23,79 \pm 2,12$ & $29,27 \pm 2,43$ \\
5/8CN & $5,48 \pm 0,56$ & $24,32 \pm 2,12$ & $29,57 \pm 2,44$ \\
5/8NC & $5,25 \pm 0,56$ & $24,05 \pm 1,51$ & $29,42 \pm 1,73$ \\
G3 & $5,37 \pm 0,40$ & \\
\hline
\end{tabular}

Diferença ${ }^{1}$

Difference $^{1}$

\begin{tabular}{|c|c|c|c|}
\hline $\mathrm{PC}-\mathrm{PN}$ & 0,32 & $-0,71$ & $-0,39$ \\
\hline & \multicolumn{3}{|c|}{$\begin{array}{l}\% \text { do peso de abate } \\
\% \text { of slaughter weight }\end{array}$} \\
\hline $3 / 4 \mathrm{CN}$ & $1,02 \pm 0,11$ & $4,09 \pm 0,33$ & $5,11 \pm 0,39$ \\
\hline $3 / 4 \mathrm{NC}$ & $0,93 \pm 0,12$ & $4,29 \pm 0,35$ & $5,22 \pm 0,41$ \\
\hline G2 & $0,97 \pm 0,08$ & $4,19 \pm 0,23$ & $5,16 \pm 0,27$ \\
\hline $5 / 8 \mathrm{CN}$ & $1,03 \pm 0,11$ & $4,55 \pm 0,32$ & $5,58 \pm 0,38$ \\
\hline $5 / 8 \mathrm{NC}$ & $1,00 \pm 0,11$ & $4,55 \pm 0,32$ & $5,55 \pm 0,38$ \\
\hline G3 & $1,02 \pm 0,08$ & $4,55 \pm 0,23$ & $5,57 \pm 0,27$ \\
\hline
\end{tabular}

Diferença ${ }^{1}$

Difference $^{1}$

\begin{tabular}{lccc} 
PC - PN & 0,06 & $-0,10$ & $-0,04$ \\
\hline \multicolumn{3}{c}{$\begin{array}{c}\% \text { do corpo vazio } \\
\% \text { of empty body }\end{array}$} \\
\cline { 2 - 4 } $3 / 4 \mathrm{CN}$ & $1,23 \pm 0,12$ & $5,01 \pm 0,36$ & $6,24 \pm 0,41$ \\
$3 / 4 \mathrm{NC}$ & $1,13 \pm 0,13$ & $5,19 \pm 0,38$ & $6,32 \pm 0,43$ \\
$\mathrm{G} 2$ & $1,88 \pm 0,08$ & $5,10 \pm 0,25$ & $6,28 \pm 0,28$ \\
$5 / 8 \mathrm{CN}$ & $1,27 \pm 0,12$ & $5,61 \pm 0,35$ & $6,88 \pm 0,40$ \\
$5 / 8 \mathrm{NC}$ & $1,23 \pm 0,12$ & $5,59 \pm 0,36$ & $6,82 \pm 0,40$ \\
$\mathrm{G} 3$ & $1,25 \pm 0,08$ & $5,60 \pm 0,25$ & $6,85 \pm 0,28$ \\
\hline
\end{tabular}

Diferença ${ }^{1}$

Difference $^{1}$

$\mathrm{PC}-\mathrm{PN}$

0,07

$-0,08$

$-0,01$

$(P>0,10) ;{ }^{1}$ Contraste entre predominância $P C(3 / 4 C N+5 / 8 C N)$ e $P N$ $(3 / 4 \mathrm{NC}+5 / 8 \mathrm{NC})$

$(P>0.10) ;{ }^{1}:$ Contrast between $P C(3 / 4 C N+5 / 8 C N)$ and $P N(3 / 4 N C+5 / 8 N C)$ predominance.

Charolês foram $0,99 \mathrm{~kg}$ superiores aos predominantemente Nelores. Menezes et al. (2004), em novilhos jovens (24 meses de idade) Charolês e Nelore, puros, terminados em confinamento, constataram maior desenvolvimento da cabeça nos novilhos Charolês. Fernandes et al. (2002) observaram maior tamanho relativo da cabeça nos animais 1/2Caracu-1/2Nelore em comparação aos 1/2Holandês1/2Nelore e Nelore puros. Galvão et al. (1991b), no entanto, não observaram aumento do tamanho de cabeça ao incluírem animais Bos taurus no cruzamento com Nelore.
Os animais 5/8NC apresentam maior desenvolvimento do úbere em comparação aos $5 / 8 \mathrm{CN}(4,21$ vs $3,07 \mathrm{~kg})$, tendência que persistiu quando corrigido para PA e PCV, acompanhando o comportamento da quantidade de gordura total descartada nesses genótipos (Tabela 7).

Não houve diferença entre grupos genéticos para peso de couro, provavelmente em virtude da similaridade do peso de abate. Peso de couro similar entre novilhos das raças Brahman (40,9 kg) e Hereford (40,2 kg) abatidos com pesos semelhantes foram verificados por Ferrel \& Jenkins (1998). Todavia, no trabalho de Galvão et al. (1991b), os animais F1 (NM e NL), em comparação aos N, apresentaram menor participação de couro no PCV. Fernandes et al. (2002) não observaram diferença no percentual de couro no PCV para novilhos $1 / 2 \mathrm{Caracu}-$ $1 / 2 \mathrm{~N}, 1 / 2$ Holandês- $1 / 2 \mathrm{~N}$ e $\mathrm{N}$ abatidos com 450 e $480 \mathrm{~kg}$ de $\mathrm{PV}$. No entanto, quando esta variável foi avaliada entre gerações de cruzamento, os animais da G3 apresentaram valores superiores em relação aos da G2 em todas as formas de expressão, resultado atribuído ao aumento do grau de heterozigose.

A gordura localizada na cavidade interna e a gordura de toalete apresentam elevado custo de produção; trata-se de um tecido de deposição tardia, de baixa eficiência energética e que demanda maior quantidade de energia para sua formação em comparação ao tecido muscular (Thompson et al., 1983; Solis et al., 1988). Neste estudo, estas características não diferiram significativamente entre as gerações de cruzamento e os grupos genéticos estudados.

\section{Conclusões}

O aumento do peso de abate de vacas de descarte resulta concomitantemente em elevação do peso de corpo vazio, porém sem alteração do rendimento de corpo vazio. Como observado para o peso do corpo vazio, os peso de cabeça e de úbere também tiveram maior desenvolvimento com o incremento do peso de abate das vacas.

A participação relativa nos pesos de abate e de corpo vazio dos componentes externos é menor em vacas mais pesadas.

A gordura total descartada acompanha o desenvolvimento do corpo vazio, demonstrando o potencial de ganho corporal energético das vacas de descarte com o aumento do peso.

Maior participação de Charolês no genótipo confere maior desenvolvimento da cabeça. Vacas da terceira geração do cruzamento rotativo Charolês $\times$ Nelore são superiores para esta característica. 


\section{Literatura Citada}

ALMeidA, M.I.V.; FOnTES, C.A.A.; AlmeidA, F.Q. et al. Conteúdo corporal e exigências líquidas de energia e proteína de novilhos mestiços Holandês-Gir em ganho compensatório. Revista Brasileira de Zootecnia, v.30, n.1, p.205-214, 2001.

ANUALPEC. Anuário da pecuária brasileira. São Saulo: FNP Consultoria \& Agroinformativos. Topal \& Biassi Editora Gráfica. 376p., 2005.

DI MARCO, O.N. Crescimiento de vacunos para carne. Mar Del Plata, 1998. 246p.

FERNANDES, H.J.; PAULINO, M.F.; GALVÃO, R.M. et al. Crescimento de componentes corporais de três grupos genéticos nas fases de recria e terminação. Revista Brasileira de Zootecnia, v.34, n.1, p.288-296, 2005.

FERRELL, C.L.; JENKINS, T.G. Body composition and energy utilization by steers of diverse genotypes fed a high-concentrate diet during the finishing period: II. Angus, Boran, Brahman, Hereford, and Tuli Sires. Journal of Animal Science, v.76, p.647-657, 1998.

GALVÃO, J.G.; FONTES, C.A.A.; PIRES, C.C. et al. Ganho de peso, consumo e conversão alimentar em bovinos não-castrados, de três grupos raciais, abatidos em diferentes estágios de maturidade (Estudo I). Revista da Sociedade Brasileira de Zootecnia, v.20, n.5, p.494-501, 1991a.

GALVÃO, J.G.; FONTES, C.A.A.; PIRES, C.C. et al. Característica e composição física da carcaça de bovinos não-castrados, abatidos em diferentes estágios de maturidade, de três grupos raciais (Estudo II). Revista da Sociedade Brasileira de Zootecnia, v.20, n.5, p.502-512, 1991 b.

JESSÉ, G.W.; THOMPSON, G.B.; CLARK, J.L. et al. Effects of ration energy and slaughter weight on composition of empty body and carcass gain of beef cattle. Journal of Animal Science, v.43, n.2, p.418-425, 1976.

JONES, S.D.M.; ROMPALA, R.E.; JEREMIAH, L.E. Growth and composition of the empty body in steers of different maturity types fed concentrate or forage diets. Journal of Animal Science, v.60, n.2, p.427-433, 1985.

JORGE, A.M.; FONTES, C.A.A. Desenvolvimento relativo das partes do corpo de zebuínos de quatro raças. Ciência Rural, v.31, n.5, p.857-861, 2001.

KUSS, F.; RESTLE, J.; BRONDANI, I.L. et al. Características da carcaça de vacas de descarte de diferentes grupos genéticos terminadas em confinamento com distintos pesos. Revista Brasileira de Zootecnia, v.34, n.3, p.915-925, 2005a.

KUSS, F.; RESTLE, J.; BRONDANI, I.L. et al. Composição física da carcaça e qualidade da carne de vacas de descarte de diferentes grupos genéticos terminadas em confinamento com distintos pesos. Revista Brasileira de Zootecnia, v.34, n.4, p.12851296, 2005b.

LEME, P.R.; BOIN, C.; MARGARIDO, R.C.C. et al. Desempenho em confinamento e características de carcaça de bovinos machos de diferentes cruzamentos abatidos em três faixas de peso. Revista Brasileira de Zootecnia, v.29, n.6, p.2347-2353, 2000 (sup1.).

MENEZES, L.F.G.; RESTLE, J.; BRONDANI, I.L. et al. Características da carcaça de novilhos de gerações avançadas do cruzamento alternado das raças Charolês e Nelore, terminadas em confinamento. Revista Brasileira de Zootecnia, v.34, n.3, p.934-945, 2005.
MENEZES, L.F.G.; RESTLE, J.; BRONDANI, L.L. et al. Características das partes do corpo não integrantes da carcaça de novilhos de gerações avançadas do cruzamento rotativo entre as raças Charolês e Nelore. III - Partes externas. In: REUNIÃO ANUAL DA SOCIEDADE BRASILEIRA DE ZOOTECNIA, 41., 2004, Campo Grande. Anais... Campo Grande: Sociedade Brasileira de Zootecnia, 2004. (CD-ROM).

MOLETTA, J.L.; RESTlE, J. Desempenho em confinamento de novilhos de diferentes grupos genéticos. Ciência Rural, v.22, n.2, p.227-233, 1992.

PEROBELLI, Z.V.; RESTLE, J.; MÜLLER, L. Estudo das carcaças de vacas de descarte das raças Charolês e Nelore. Pesquisa Agropecuária Brasileira, v.30, n.3, p.409-412, 1995.

RESTLE J. Comportamento reprodutivo do rebanho de gado de corte da fazenda experimental de criação experimental agronômica da UFRGS. $1^{\circ}$ Semestre. Seminário da disciplina de Técnicas de Pesquisas. Curso de PósGraduação em Agronomia, Universidade Federal do Rio Grande do Sul, 1972.

RESTLE, J.; MENEZES, L.F.G.; ARBOITTE, M.Z. et al. Características das partes não-integrante da carcaça de novilhos 5/6 Nelore 3/8 Charolês abatidos em três estádios de desenvolvimento. Revista Brasileira de Zootecnia, v.34, n.4, p.1339-1348, 2005.

RESTLE, J.; PASCOAL, L.L.; FATURI, C. et al. Efeito do grupo genético e da heterose nas características quantitativas da carcaça de vacas de descarte terminadas em confinamento. Revista Brasileira de Zootecnia, v.31, n.1, p.350-362, 2002 (supl.).

RESTLE, J.; QUADROS, A.R.B.; VAZ, F.N. Terminação em confinamento de novilhos de diferentes genótipos Hereford $\mathrm{x}$ Nelore. Revista Brasileira de Zootecnia, v.29, n.1, p.125130,2000 .

SANTOS, A.P.; BRONDANI, I.L.; RESTLE, J. et al. Influência do grupo genético e da dieta alimentar no peso do corpo vazio e órgãos vitais de novilhos superprecoce. In: REUNIÃO ANUAL DA SOCIEDADE BRASILEIRA DE ZOOTECNIA, 40., 2003 , Santa Maria. Anais... Santa Maria: Sociedade Brasileira de Zootecnia, 2003. (CD-ROM).

STATISTICAL ANALYSES SYSTEM - SAS. SAS/STAT user's guide statistics. 4.ed. Cary: 1997, v.2, 943p.

SIGNORETTI, R.D.; ARAÚJO, G.G.L.; SILVA, J.F.C. et al. Características quantitativas da partes do corpo não-integrante da carcaça animal e desenvolvimento do trato gastrintestinal e bezerros da raça holandesa alimentados com dietas contendo quatro níveis de concentrado. Revista Brasileira de Zootecnia, v.28, n.4, p.875-882, 1999.

SOLIS, J.C.; BYERS, F.M.; SCHELLING, G.T. et al. Maintenance requirements and energetic efficiency of cows of different breed types. Journal of Animal Science, v.66, p.764-773, 1988.

THOMPSON, W.R.; MEISKE, J.C.; GOODRICH, R.D. et al. Influence of body composition on energy requirements of beef cows during winter. Journal of Animal Science, v.56, n.5, p.1241-1252, 1983. 